In Cres. Vol. $3 N^{\circ}$ 1: pp. 109-119, 2012

\title{
COMUNICACIÓN FAMILIAR Y FORMAS DE USO DE LA TELEVISIÓN E INTERNET EN LAS FAMILIAS*
}

\author{
FAMILY COMMUNICATION AND METHODS OF \\ USING THE INTERNET AND THE TELEVISION \\ IN THE FAMILIES
}

Fanny Rocío Nique Tapia ${ }^{1}$, Carmen Gorritti Siappo ${ }^{2}$

\begin{abstract}
RESUMEN
La presente investigación cuantitativa, de diseño descriptivo correlacional no experimental, buscó establecer la relación entre el nivel de comunicación familiar y las formas de uso de la televisión e internet, en familias del A.H. 25 de Mayo de la ciudad de Chimbote. El universo muestral estuvo constituido por 64 familias que cumplieron con los criterios de inclusión. Para la recolección de datos se usó tres instrumentos: el primero es una escala de comunicación familiar, la cual nos da a conocer el nivel de comunicación familiar en la familia; el segundo test valora las formas de uso de la televisión; y el tercero, las formas de uso de internet en las familias. En el procesamiento de datos se usó la estadística descriptiva e inferencial, llegando a las siguientes conclusiones: La mayoría de las familias del A.H. 25 de Mayo de Chimbote, presentan nivel de comunicación adecuado; sin embargo existe un porcentaje importante que presenta nivel de comunicación inadecuado. La mayoría presentan forma de uso inadecuado de la televisión. Existe relación estadísticamente significativa entre el nivel de comunicación familiar y la forma de uso de la televisión en las familias del A.H. 25 de Mayo. La comunicación familiar y uso del internet presentan relación estadísticamente significativa en las familias del A.H. 25 de Mayo de Chimbote.

PALABRAS CLAVE: Familia, Comunicación familiar, Medios de comunicación, Televisión e Internet.
\end{abstract}

* Recibido: 20 de enero del 2012; aceptado: 14 de junio del 2012.

1 Maestra en Enfermería, Mención: Salud Pública. Docente de la Escuela de Enfermería. Universidad Católica Los Ángeles de Chimbote.

2 Maestra en Enfermería, Mención: Salud familiar y comunitaria. Directora de la Escuela Profesional de Enfermería. Universidad Católica Los Ángeles de Chimbote. 


\begin{abstract}
This nonexperimental research of descriptive correlational design aimed to establish the relationship between the level of family communication and the use of television and the internet in families of the shanty town (Asentamiento Humano) 25 de Mayo of Chimbote city. The sample population consisted of 64 families. Three were the data collection instruments used; The Family Communication Scale, The Television Use Questionnaire and the Internet Use Questionnaire. The descriptive and inferential statistics was used to data collection. The study came to the following conclusions: Most of the families of the shanty town 25 de Mayo have adequate communication level, but there is a significant percentage of communication that has inadequate level. Most evidence inappropriate use of television. There is a statistically significant relationship between the level of family communication and the way the television is used. The family communication and the use of internet have a statistically significant relationship in the families of the shanty town 25 de Mayo.
\end{abstract}

KEY WORDS: Family, family communication, media, television and internet.

\title{
I. INTRODUCCIÓN
}

En las relaciones humanas resulta imposible no comunicarse, ya que el comportamiento humano se basa en el intercambio de información y en la comunicación. Hace unas décadas un proceso importante se inició con la digitalización de las comunicaciones humanas, el mismo que ha ido acelerando a medida que evoluciona la tecnología. ${ }^{1}$

El nuevo siglo enfrenta al Perú y al mundo a la era de la información, cuya característica fundamental es posibilitar el establecimiento de una sociedad basada en el acceso a la información y al conocimiento, una sociedad denominada Sociedad de la Información, la que para su desarrollo necesita del despliegue y uso intensivo de tecnologías de información y comunicaciones (TIC). ${ }^{2}$

La familia es la primera escuela donde se aprende cómo comunicarse y la forma cómo se aprende a comunicarse en la familia de origen, determinará cómo comunicarse con los demás. El niño comienza aprendiendo gestos y tonos de voz de sus padres y hermanos, comunicándose a través de ellos. Por ejemplo, cuando señala con el dedo y pide "ete" ó "quielle" para pedir algo. En este caso, es la familia la que entiende e interpreta lo que quiere decir.,

La forma de comunicarse que tienen los miembros de la familia determinará la forma en que los niños que en ella crecen aprendan una manera de emocionarse y de pensar. Esto significa que cada familia enseña a través de la forma que tiene de comunicarse, su estilo particular; los valores, forma de pensar y mirar el mundo. ${ }^{5,4}$ 
Ahora bien, no es sólo cuestión de tener en cuenta cómo los medios de comunicación influyen en las familias y el contexto en el cual éstas se desenvuelven, sino también contemplar la otra perspectiva; es decir, cómo los medios de comunicación pueden influenciar en el comportamiento existente dentro de las familias y ante ello la familia puede desempeñar un verdadero papel para el aprendizaje y la concreción de los medios. Los efectos pueden variar para algunos niños; bajo ciertas condiciones, son perjudiciales; para otros niños bajo idénticas condiciones, puede ser beneficioso. ${ }^{6,7,8}$

Ningún medio en la historia de la humanidad se ha introducido con tanta rapidez y fuerza como lo está haciendo Internet. El número de usuarios crece de forma tan exponencial, que se encuentra con problemas de saturación de la red y con la creación de nuevas redes como Internet para usos académicos y de investigación. Es importante señalar que la era digital en nuestro país no se define tanto por la propiedad sobre las computadoras, sino por el acceso a la red informática, por lo que en este caso el capital más importante sería el aprendizaje, y no la posesión de un equipo. En el Perú el uso de Internet se está difundiendo de este modo, no por la tenencia de computadoras en el hogar, sino por el uso de cabinas públicas. ${ }^{9,10}$

En el informe de la Universidad Pontificia Católica del Perú, según los cabineros, el interés fundamental al usar Internet es el entretenimiento y en este ámbito, las páginas sobre espectáculos, música y pornografía son las más visitadas. Pese a la evidencia (historial de uso de las computadoras), el interés por la pornografía no es mencionado por los usuarios en sus entrevistas (se registra un solo caso). El tema se oculta y se evade una y otra vez, pese a que todas las cifras acerca del consumo de Internet demuestran lo contrario. La pornografía es un tema muy frecuentemente dentro del uso de Internet; gran cantidad de usuarios se dedican a esto en su tiempo en línea. ${ }^{11}$

\section{PROBLEMA}

¿Cuál es la relación entre el nivel de comunicación familiar y las formas de uso de la televisión y el internet en las familias del A.H. 25 de Mayo, de Chimbote, 2008 ?

\section{OBJETIVOS}

\section{Objetivo general}

Establecer la relación que existe entre el nivel de comunicación familiar y las formas del uso de la televisión e internet en las familias del A.H. 25 de Mayo. 


\section{Objetivos específicos}

a. Determinar el nivel de comunicación familiar en el A.H. 25 de Mayo.

b. Valorar las formas de uso de la televisión en las familias del A.H. 25 de Mayo.

c. Valorar las formas de uso del internet en las familias del A.H. 25 de Mayo.

Con el enfoque de complejidad: "familia es un organismo vivo complejo, cuya trayectoria de vida es un transcurrir de diversidades, adversidades, semejanzas, diferencias, individualidades, singularidades y complementariedades, que lucha por su preservación y desarrollo en un tiempo, espacio y territorio dados y al cual se siente perteneciente, interconectada y enraizada biológica, solidaria, amorosa, cultural, política y socialmente". ${ }^{2}$

No puede existir una familia, ni se puede lograr la unidad de la misma sin la adecuada comunicación entre sus miembros. La teoría de la comunicación es un campo de estudio dentro de las sociedades que trata de explicar cómo se realizan los intercambios comunicativos y cómo estos intercambios afectan a sociedad y comunicación. Es decir, investiga el conjunto de principios, conceptos y regularidades que sirven de base al estudio de la comunicación como proceso social. Está en estrecha relación con otras ciencias, de las cuales toma parte de sus contenidos o los integra entre sí. ${ }^{10}$

La comunicación es un fenómeno inherente a la relación grupal de los seres vivos, por medio del cual éstos obtienen información acerca de su entorno y son capaces de compartirla haciendo partícipes a otros de esa información. Siendo un proceso de interrelación entre dos (o más) personas, donde se transmite una información desde un emisor que es capaz de codificarla en un código definido hasta un receptor, el cual decodifica la información recibida. El proceso de comunicación emisor-mensaje-receptor, se torna bivalente cuando el receptor logra codifica el mensaje, lo interpreta y lo devuelve al emisor originario, quien ahora se tornará receptor. ${ }^{12}$

Las bases para una comunicación efectiva y sana dentro del vínculo familiar se debe trabajar. Muchas veces encontramos en las familias temas que están vedados, mitos, rituales, secretos familiares. En la familia, una mala comunicación puede dificultar las relaciones entre los padres y los hijos. Es común escuchar adolescentes que afirman que sus padres no entienden o a padres decir que sus hijos no les hacen caso. En ocasiones pareciera que dentro de una misma 
familia todos hablan lenguajes diferentes. Cuando la comunicación tiene como objetivo el entendimiento mutuo, la vida social tiene mejores pronósticos. ${ }^{13}$

La palabra "televisión" es un híbrido de la voz griega "Tele" (distancia) y la latina "visio" (visión). El término televisión se refiere a todos los aspectos de transmisión y programación de televisión. Esta transmisión puede ser efectuada mediante ondas de radio o por redes especializadas de televisión por cable. El receptor de las señales es el televisor. ${ }^{14}$

Internet es un método de interconexión de redes de computadoras implementado en un conjunto de protocolos denominado TCP/IP y garantiza que redes físicas heterogéneas funcionen como una red (lógica) única. Hace su aparición por primera vez en 1969, cuando ARPA net establece su primera conexión entre tres universidades en California y una Utah. Ha tenido la mayor expansión en relación a su corta edad, comparada por la extensión de este medio. Su presencia en casi todo el mundo, hace de la Internet un medio masivo, donde cada uno puede informarse de diversos temas en las ediciones digitales de los periódicos, o escribir según sus ideas en bloqs y fotobloq, o subir material audiovisual como en el popular sitio Youtube. ${ }^{15}$

La llegada de Internet no sólo tuvo un impacto importante en los medios tradicionales, sino también en el fortalecimiento de los medios alternativos y comunitarios como la radio, la televisión y la prensa comunitaria, favoreciendo procesos sociales de comunicación interactiva e intercambios en multimedia, producto de la digitalización de los mensajes y de la integración de los "telecentros". Internet abrió la posibilidad de un espacio inédito de intercambio de la información fuera de los circuitos de los conglomerados mediáticos, lo que contribuyó a dar una dimensión real al movimiento social mundial de la sociedad civil en temas globales. ${ }^{15,16}$

Esta situación presenta una relación entre dos variables que, pensamos, están condenadas a entenderse por el buen funcionamiento de la sociedad. El binomio familia-medios de comunicación, o lo que es lo mismo, medios de comunicación-familia, es un matrimonio de difícil disolución, cuya relación -aún hoy- puede y debe ser mejorada e incentivada. Juntos pueden ayudar a que la sociedad del siglo XXI mejore y avance de forma vertiginosa alejándola de turbulencias. La tarea no es fácil y necesita el beneplácito de ambas partes, e incluso la participación de terceros, como pueden ser los centros educativos. ${ }^{17}$

La complejidad del enlace familia-medios/medios-familia, debido a que los medios han contribuido a la modificación de las relaciones personales, han potenciado la inactividad y la generación de necesidades, las cuales muchas 
veces ni siquiera existen en la mente del sujeto, cubren deseos ficticios, favorecen la imaginación, -llegando incluso a producir conductas obsesivas-, potencian conductas negativas. El influjo que "sufren" los miembros de las familias desde los MCM ha mediatizado el conocimiento que los individuos poseen de la realidad, de los hábitos y costumbres de toda la comunidad. Ante tal circunstancia, la familia debe actuar de forma contundente..$^{17,18}$

\section{METODOLOGÍA}

El presente estudio de investigación es de tipo cuantitativo, de corte transversal, descriptivo, correlacional. El diseño es descriptivo correlacional. ${ }^{19,20}$ La población muestral estuvo constituida por 64 familias de la jurisdicción del A.H. 25 de Mayo, del distrito de Chimbote.

La variable Nivel de comunicación familiar está definida como la capacidad que tienen los miembros de la familia para relacionarse entre sí; a través de ella se conocen y se negocian los espacios en la vida cotidiana, al igual que se entregan o vivencian las creencias, las costumbres y los estilos de vida propios de cada familia. ${ }^{1}$ La variable Formas de uso de la televisión, referida a la interacción que tiene la familia con la televisión relacionada a su cantidad, ubicación en la vivienda, programación y control de la misma. ${ }^{17}$

La variable Formas de uso de Internet referido a la interacción que tiene la familia con método de interconexión de redes de computadoras llamado "internet”. Dicha relación, referida a acceso, miembros de la familia que lo usan, frecuencia de uso, control y motivos de uso. ${ }^{15}$

Los instrumentos fueron validados por tres expertos que acreditaron experiencia en el área, dando las sugerencias pertinentes para mejorar los instrumentos. Para el procesamiento de datos se usó la estadística descriptiva e inferencial. Los resultados obtenidos se presentaron en cuadros simples y de doble entrada y se elaboró gráfico para cada cuadro, empleando el software especializado de estadística, paquete SPSS, versión 15.0. Para establecer la relación entre las variables se utilizó el Chi cuadrado con el $95 \%$ de confianza.

\section{RESULTADOS Y DISCUSIÓN}

TABLA 01: Nivel de comunicación familiar de las familias del A.H. 25 de Mayo, Chimbote, 2008. Se evidencia que en su mayoría el nivel es adecuado, con un $71,9 \%$ y un $28,1 \%$ es inadecuada.

TABLA 02: Forma de uso de la televisión en las familias del A.H. 25 de 
Mayo, Chimbote, 2008. Muestra que 59,4\% usa la TV adecuadamente y el $40,6 \%$, inadecuadamente.

TABLA 03: Forma de uso de internet en las familias del A.H. 25 de Mayo, Chimbote, 2008. Muestra la forma de uso de internet en las familias del AA.H H. 25 de Mayo, en donde la forma de uso adecuado obtiene el menor porcentaje, un $9,4 \%$, la forma de uso inadecuado comprende un $39,1 \%$, así mismo que la mayoría de las familias en estudio no hacen uso del medio, ello con un 51,6\%.

Tabla 01

NIVEL DE COMUNICACION FAMILIAR DE LAS FAMILIAS DEL

A.H. 25 DE MAYO, CHIMBOTE, 2008

\begin{tabular}{llc}
\hline Nivel de comunicación familiar & $\mathbf{N}^{\circ}$ & $\%$ \\
\hline Adecuada & 46 & 71,9 \\
Inadecuada & 18 & 21,1 \\
\hline Total & 64 & 100,0 \\
\hline
\end{tabular}

Fuente: Escala de comunicación familiar. Elaborado por Ñique F. Aplicado a las familias del A.H. 25 de Mayo - Chimbote - 2008.

Tabla 02

FORMA DE USO DE LA TELEVISION EN LAS FAMILIAS

DEL A.H. 25 DE MAYO

\begin{tabular}{lcc}
\hline Formas de uso de la TV & $\mathbf{N}^{\circ}$ & \% \\
\hline Adecuado & 38 & 59,4 \\
Inadecuado & 26 & 40,6 \\
\hline Total & 64 & 100,0 \\
\hline
\end{tabular}

Fuente: Cuestionario sobre formas de uso de la TV. Elaborado por Ñique F. Aplicado a las familias del A.H. 25 de Mayo - Chimbote - 2008.

Tabla 03

FORMA DE USO DE INTERNET EN LAS FAMILIAS

DEL A.H. 25 DE MAYO

\begin{tabular}{llc}
\hline Formas de uso de Internet & $\mathbf{N}^{\circ}$ & \% \\
\hline Adecuado & 06 & 9,4 \\
Inadecuado & 25 & 39,1 \\
No hace uso del medio & 33 & 51,6 \\
\hline Total & 64 & 100,0 \\
\hline
\end{tabular}

Fuente: Cuestionario sobre formas de uso del internet. Elaborado por Nique F. Aplicado a las familias del A.H. 25 de Mayo - Chimbote - 2008. 
Tabla 04

NIVEL DE COMUNICACIÓN FAMILIAR SEGÚN LA FORMA DE USO DE LA TELEVISIÓN EN LAS FAMILIAS

\begin{tabular}{|c|c|c|c|c|c|c|}
\hline \multirow{3}{*}{$\begin{array}{l}\text { Nivel de } \\
\text { comunicación } \\
\text { familiar }\end{array}$} & \multicolumn{4}{|c|}{ Forma de uso de la TV } & & \\
\hline & \multicolumn{2}{|c|}{ Adecuado } & \multicolumn{2}{|c|}{ Inadecuado } & \multicolumn{2}{|c|}{ Total } \\
\hline & $\mathbf{N}^{\circ}$ & $\%$ & $\mathbf{N}^{\circ}$ & $\%$ & $\mathbf{N}^{\circ}$ & $\%$ \\
\hline Adecuado & 24 & 37,5 & 22 & 34,4 & 46 & 71,9 \\
\hline Inadecuado & 02 & 3,1 & 16 & 25,0 & 18 & 28,1 \\
\hline TOTAL & 26 & 40,6 & 38 & 59,4 & 64 & 100,0 \\
\hline
\end{tabular}

Fuente: Escala de comunicación familiar y Cuestionario sobre formas de uso de la TV, elaborado por Ñique F., aplicado a las familias del A.H. 25 de Mayo - Chimbote - 2008.

$$
\mathrm{x}^{2}=0,14 \quad \mathrm{P}<0,05
$$

Tabla 05

NIVEL DE COMUNICACIÓN FAMILIAR SEGÚN LA FORMA DE USO DE INTERNET EN LAS FAMILIAS

\begin{tabular}{|c|c|c|c|c|c|c|c|c|}
\hline \multirow{3}{*}{$\begin{array}{l}\text { Nivel de } \\
\text { comunicación } \\
\text { familiar }\end{array}$} & \multicolumn{6}{|c|}{ Forma de uso de internet } & & \\
\hline & \multicolumn{2}{|c|}{ Adecuado } & \multicolumn{2}{|c|}{ Inadecuado } & \multicolumn{2}{|c|}{ No usa el medio } & \multicolumn{2}{|c|}{ Total } \\
\hline & $\mathbf{N}^{\circ}$ & $\%$ & $\mathbf{N}^{\circ}$ & $\%$ & $\mathbf{N}^{\circ}$ & $\%$ & $\mathbf{N}^{\circ}$ & $\%$ \\
\hline Adecuado & 05 & 7,9 & 14 & 21,9 & 27 & 42,1 & 46 & 71,9 \\
\hline Inadecuado & 01 & 1,5 & 11 & 17,2 & 06 & 9,4 & 18 & 28,1 \\
\hline TOTAL & 06 & 9,4 & 25 & 39,1 & 33 & 51,6 & 64 & 100,0 \\
\hline
\end{tabular}

Fuente: Escala de comunicación familiar y Cuestionario sobre formas de uso del internet. Elaborado por Ñique F., aplicado a las familias del A.H 25 de Mayo - Chimbote - 2008.

$$
\mathrm{x}^{2}=0,12 \mathrm{P}<0,05
$$

TABLA 04: Nivel de comunicación familiar según la forma de uso de la televisión en las familias del A.H. 25 de Mayo, Chimbote, 2008. Se observa que las familias que tienen un nivel de comunicación adecuado $(71,9 \%)$ presentan un $37,5 \%$ de uso adecuado de la televisión y un $34,4 \%$ una forma de uso inadecuado del medio; así mismo, las familias que tienen un nivel de comunicación inadecuado $(28,1 \%)$ presentan en su mayoría una forma inadecuada del uso de la televisión, evidenciado por un $25 \%$ del total de familias en estudio.

TABLA 05: Nivel de comunicación familiar según la forma de uso del internet en las familias del A.H. 25 de Mayo, Chimbote, 2008. Muestra la distribución de la relación existente entre el nivel de comunicación familiar y 
la forma de uso del internet, en donde las familias que tienen un nivel de comunicación adecuado $(71,9 \%)$ en su mayoría no hacen uso del medio con un $42,1 \%$, un $21,9 \%$ de uso inadecuado del medio y un $7,9 \%$ una forma de uso adecuado del medio; así mismo, las familias que tienen un nivel de comunicación inadecuado $(28,1 \%)$ presentan un $17,2 \%$ de una forma inadecuada del uso del internet, un $9,4 \%$ que no usa el medio y un $1,5 \%$ que lo usa adecuadamente.

Según la evidencia de la investigación, el nivel de comunicación adecuado puede explicarse, al menos en parte, atendiendo a la diferente implicación que los padres (sobre todo la madre) tienen en la crianza y educación de sus hijos e hijas. En la muestra en estudio, las madres parecen estar más presentes en el hogar que los padres, y no sólo debido a que la frecuencia del trabajo extradoméstico es menor para las mujeres, sumado a ello que al tener que trabajar no tienen quién quede en casa cuidando a los niños, sino que incluso las madres que trabajan fuera del hogar están más implicadas en las vidas de sus hijos e hijas.

Blas, B y col. ${ }^{21}$ comenta: "La televisión hoy forma parte del mobiliario de todos los hogares. Sin embargo, no se trata de un mueble más; si así fuere sería fácil prescindir de ella o relegarla a un segundo lugar. No, lamentablemente la televisión se ha convertido en el centro de la familia y se alza en el cuarto de estar como si se tratara de un altar ante el que todos nos inclináramos para pedirle dones o entregarle nuestras ofrendas. Creemos que no nos equivocamos al afirmar que la televisión se ha convertido en el centro de la vida social de la familia; en torno a ella se unen sus miembros, y es ella la que tiende a seleccionar los contenidos de discusión y las actividades a la que son dedicadas las horas libre de ocio.

Los resultados obtenidos son similares en cuanto al uso a internet con los estudios realizados por el INEI, en donde nos dice que un 43,5\% hacen uso de internet en la zona urbana de Ancash. Es importante señalar que la era digital en el país no se define tanto por la propiedad sobre las computadoras, sino por el acceso a la red informática, por lo que en este caso el capital más importante sería el aprendizaje, y no la posesión de un equipo. En el país, el uso de Internet se está difundiendo de este modo, no por la tenencia de computadoras en el hogar, sino por el uso de las cabinas públicas. ${ }^{2}$ El nivel de comunicación familiar y las formas de uso de la televisión son variables que se relacionan significativamente, evidenciado ello al aplicar la prueba de independencia de criterios chi cuadrado.

Los resultados encontrados guardan relación con los estudios de Blas y Cabero, ${ }^{22}$ quienes afirman que "se ha encontrado una alta correlación positiva 
en las investigaciones que se han centrado en estos estudios de comportamientos violentos, entre los programas que observaban los adolescentes y el comportamiento agresivo de la familia. Ello posiblemente lleva a asumir que algunas veces, y bajo ciertas condiciones, los medios más que crear patrones culturales, lo que sirven son como elementos potenciadores de patrones culturales y sociales ya asumidos conscientes o inconscientemente en los entornos familiares".

Los medios de comunicación, especialmente la televisión, son un factor importante en la definición, la estructura y la organización de las familias. La televisión ofrece modelos de comportamiento para las familias, respecto a los roles, los valores y las normas. Los medios de comunicación contribuyen a dar forma a los ritmos de la vida familiar. La televisión, los periódicos y la radio, e incluso la internet, forman parte de las rutinas diarias de muchos adultos y niños, así como la música, los libros y los vídeos son importantes en la vida cotidiana de los niños. La propia realidad social de la familia se construye en parte alrededor de esos medios y tecnologías. ${ }^{17}$

Por tanto, los medios de comunicación han obtenido un espacio importante en la vida de las personas, por lo que es relevante prestar atención a la relación entre los medios de comunicación, como la televisión e internet y la familia; por ser el hogar el ámbito donde el niño inicia y accede a diversos medios de comunicación de masas (televisión, Internet, video juegos, etc.), y es precisamente en este espacio, donde los padres, en comunicación con sus hijos, deben desarrollar un papel mediador en el establecimiento de los criterios que deben regular el uso del mismo.

\section{CONCLUSIONES}

1. La mayoría de familias del A.H. 25 de Mayo, de Chimbote, presenta un nivel de comunicación adecuado, y un porcentaje importante presenta nivel de comunicación inadecuada.

2. Más de la mitad de familias del A.H. 25 de Mayo presentan una forma de uso inadecuado de la televisión.

3. Un poco más de la mitad las familias del A.H. 25 de Mayo no hacen uso de internet y menos de la mitad lo usan de manera inadecuada.

4. Existe relación estadísticamente significativa entre el nivel de comunicación familiar y la forma de uso de la televisión en las familias del A.H. 25 de Mayo.

5. Las variables de comunicación familiar y uso del internet presentan una relación significativa en las familias del A.H. 25 de Mayo, de Chimbote. 


\section{REFERENCIAS BIBLIOGRÁFICAS}

1 ENRíQueZ R., Y MAGAÑa RUIZ, R. Comunicación familiar, autoestima y calidad de vida. España. 2006.(citada en 2009, marzo) Disponible en: http://148.201.94.3:8991/F?func=direct\&current base $=$ ITE01\&doc_number $=000044516$.

2 INEI. Tecnologías de Información y Comunicaciones en los Hogares Lima, Dirección Técnica de Demografía e Indicadores Sociales. ENAHO 2003/2004. Perú - Lima .2005 Bustamente E, Enfermería familiar. Universidad Nacional de Trujillo. Perú. 2004.

3 MARÍn Y LATORRE M. Los medios de comunicación y la familia ¿un matrimonio de conveniencia?. Edutec. Asociación para el Desarrollo de la Tecnología Educativa, No 21. ISSN:1135-9250. Mallorca, España. En Revista Realidad. Año X, N², 2005.

4 CARrillo G. T. La comunicación Familiar. 2008 (citada en 2009, marzo). Disponible en: http://www.renuevodeplenitud.com/mejorando-la-comunicacion-en-la-familia.html.

5 BERMEJO y CABERO, J. Familia y medios de comunicación. Medios de comunicación y familia. Universidad de Sevilla. 2004.

6 WRIGHT, N. Claves para una mejor comunicación en el matrimonio. Editorial Unilit: Colombia. 1994.

7 CABERO, A. Familia y medios de comunicación. Medios de comunicación y familia. Universidad de Sevilla. 2da. Edición. 2004.

8 Neghme E. V. Medios de comunicación y Valores democráticos. 2003. (citada en 2009, marzo).

9 Loyola, Morales, Vidal, E. Los consumos de medios de comunicación en los mayores de 50 años: entre el asombro y la nostalgia (6.313 palabras - 13 páginas) Universidad Nacional de Córdoba Argentina.1998 (citada en 2009, marzo). Disponible en: http://www.lazarillo.com/ latina/a/11loyolaco.htm.

10 Colona G. Las Cabinas Públicas de Internet en Lima: Procesos de comunicación y formas de incorporación de la tecnología a la vida cotidiana. Facultad de Ciencias y Artes de la Comunicación y Departamento de Comunicaciones Pontificia Universidad Católica del Perú. Informe de Investigación.2003.

11 Bostardos Boadas, A. Comunicación Humana y Paradigmas Holísticos. Publicación Claves de la Razón Práctica. España. 1995.

12 PARRA JimÉnez Y A. Oliva D. Comunicación y conflicto familiar durante la adolescencia. Universidad de Sevilla. España. Anales de psicología. Vol 18. 215-231.2002.

13 García, F. Los medios de comunicación al servicio de la comunidad. España. Grupo Aor. 2003.

14 GISPERT, M. Las tecnologías de la información y la Comunicación como favorecedoras de los procesos de autoaprendizaje y de formación permanente. España 1999.

15 Froufe, S. Familia y Medios de comunicación1998. (citada en 2009, marzo) Disponible en: htpp://tecnologiaeducativa.es/revista/familia.htm.

16 Cebriam, M. La televisión. Creer para ver. Málaga. Edit. Clave. 1992.

17 MARÍn, V. Y GARCíA, MA. D. La familia e Internet, un juego o tres bandas. Comunicar. Córdova España. 2003.

18 HERnÁNDEZ y FERNÁNDEZ. Metodología de la Investigación Científica. Edit. Mc GrawHill. España. 2006.

19 Potter, A. y Perry, A, Fundamentos de enfermería. Edit. Océano. España. 2003.

20 BlAS BERMEJO Y COL. Familia y medios de comunicación. Universidad de Sevilla.2002. (citada en 2009, marzo). Disponible en: http//:Tecnologiaedu.us.es/revistaslibros/familia.

21 Sapramona, J. Dimensión educativa de los medios de comunicación. Educación Familiar. Madrid. 2003. 
